\title{
Light scattering by cometary dust numerically simulated with aggregate particles consisting of identical spheres ${ }^{\star}$
}

\author{
H. Kimura ${ }^{1}$, L. Kolokolova ${ }^{2}$, and I. Mann ${ }^{3}$ \\ 1 Institute of Low Temperature Science, Hokkaido University, Kita-ku Kita-19 Nishi-8, Sapporo 060-0819, Japan \\ e-mail: hiroshi_kimura@lowtem.hokudai.ac.jp \\ 2 Planetary Data System Group, Department of Astronomy, Rm. 2337, Computer and Space Science Bldg., University of Maryland, \\ College Park, MD, 20742, USA \\ e-mail: ludmilla@astro.umd.edu \\ 3 Institut für Planetologie, Westfälische Wilhelms-Universität, Wilhelm-Klemm-Str. 10, 48149 Münster, Germany \\ e-mail: imann@uni-muenster.de
}

Received 3 August 2004 / Accepted 28 November 2005

\section{ABSTRACT}

Context. Optical characterization of dust particles in cometary comae is based on a comparison between observations and simulations of solar radiation scattered by cometary dust.

Aims. Our recent studies suggest that all the observed features can be reproduced by dust aggregates consisting of optically dark submicrometersize grains when the overall sizes of the aggregates are much larger than visible wavelengths.

Methods. To put constraints on the properties of cometary dust aggregates, we thoroughly investigate the angular and spectral dependencies of intensity and polarization for aggregate particles consisting of identical homogeneous spheres based on a rigorous light-scattering theory.

Results. The optimum parameters found through our comprehensive survey are $a_{\mathrm{V}} \gg 0.6 \mu \mathrm{m}$ for the radius of aggregates, $a_{\mathrm{m}} \approx 0.1 \mu \mathrm{m}$ for the radius of constituent spheres, $n \approx 1.8-2.0$ for the real part of the refractive index, $k \approx 0.4-0.6$ for the imaginary part and $\mathrm{d} m / \mathrm{d} \lambda \gtrsim 0$ for the spectral gradient of the refractive index. In particular, the best results are obtained for the refractive indices derived from a synthetic mixture of carbonaceous material, magnesium-rich silicate, and iron-bearing sulfide with their abundances simulating the composition of dust in the comet 1P/Halley.

Conclusions. Despite the simplicity of our model, these parameters are entirely consistent with our best knowledge of the properties of cometary dust. The common angular and spectral dependencies of intensity and polarization observed for cometary dust are explained with similarities in the average size and element composition of the constituent grains forming dust aggregates. Slight differences in the observed optical data may result from variations in the processing of cometary matter.

Key words. comets: general - interplanetary medium - meteors, meteoroids - polarization - scattering

\section{Introduction}

The physical and chemical properties of dust particles in comets provide one clue to the formation of the solar system, since comets are the relics of planetary formation. In the visible wavelength range, a number of comets have been observed to date, but it is not straightforward to infer the properties of cometary dust solely from the observational data. Laboratory simulations of solar radiation scattered by cometary dust have been carried out to better understand the properties of the dust (e.g., Giese 1980; Greenberg \& Gustafson 1981; Gustafson \& Kolokolova 1999; Muñoz et al. 2000; Hadamcik et al. 2002). In addition, computer simulations of the scattered light have been performed to construct a plausible model of

* Figures 2-4, 8 and 9 are only available in electronic form at http://www . edpsciences.org cometary dust (e.g., Mukai et al. 1987; Mukai \& Mukai 1990; Levasseur-Regourd et al. 1997; Lumme et al. 1997; Xing \& Hanner 1997; Petrova et al. 2000; Kerola \& Larson 2001; Kimura 2001; Petrova et al. 2004). These previous studies have attempted to reproduce typical dependencies of albedo and linear polarization on scattering angle and wavelength for a variety of comets ${ }^{1}$, and these common optical properties of cometary dust are listed below.

1. The albedo is a smooth function of scattering angle with a weak enhancement in the backscattering and a strong

\footnotetext{
1 Throughout this paper, the intensity of scattered solar radiation is termed albedo defined by Hanner et al. (1981) as a phase function normalized to the geometric albedo at the backscattered direction, and we use a scattering angle to describe the geometry of a comet and an observer with respect to the Sun, instead of a phase angle, which commonly appears in the literature on cometary observations.
} 
broad peak in the forward scattering (Millis et al. 1982; Gehrz \& Ney 1992). Nevertheless, the albedo of backscattered radiation is still low with its value less than 0.06 (Hanner \& Newburn 1989; Lamy et al. 1989).

2. The dependence of albedo on wavelength exhibits a red color relative to the solar continuum without significant variations at different scattering angles (Jewitt \& Meech 1986; Kolokolova et al. 1997).

3. Linear polarization shows a bell-shaped curve in relation to the scattering angle with its maximum value of typically 10-30\% (Dobrovolsky et al. 1986; Levasseur-Regourd et al. 1996). The maximum lies in a scattering angle between 80 and $90^{\circ}$, a value that may depend on comets (Kiselev \& Velichko 1998; Manset \& Bastien 2000). The degree of linear polarization becomes negative at scattering angles that are larger than $150-160^{\circ}$ with a minimum of approximately $-2 \%$ (Dollfus et al. 1988; Eaton et al. 1992).

4. The color of linear polarization is red, in other words, the polarization increases with wavelength (Chernova et al. 1996; Kolokolova \& Jockers 1997). The polarimetric color gradually approaches neutral or might even become blue towards the backscattering region (Michalsky 1981; Kikuchi et al. 1987).

It has been difficult to simultaneously account for all the lightscattering properties of cometary dust by a single model (see Kolokolova et al. 2004a,b; Mann et al. 2004). We recently found from our numerical simulations that these common characteristics of cometary dust can be qualitatively explained by large aggregates consisting of optically dark submicrometersize grains (Kimura et al. 2003; Kolokolova et al. 2004b; Mann et al. 2004). The model describes the aggregates with plausible assumptions on the configuration, number, size, and refractive index of the constituent grains (monomers). However, it has not been fully understood how each parameter influences the light-scattering properties nor has it been thoroughly explored which parameter space provides quantitative best fits to the observed angular and spectral dependences of albedo and polarization. The best set of parameters, if uniquely found, would provide a perspective to establish a comprehensive model of cometary dust that serves for interpreting not only visible but also infrared data. The main purpose of this paper is to place constraints on the model for cometary dust by making a thorough survey of the parameters. To avoid time-consuming computations with unreasonable parameters, we first constrain the parameter space using currently available information on the properties of cometary dust in Sect. 2 . We present our numerical results on albedo and polarization in Sect. 3 and discuss the results in Sect. 4.

\section{Model dust aggregates}

\subsection{The configuration of monomers}

Comets were most likely formed in the solar nebula as an agglomeration of presolar interstellar dust and partly protosolar nebular condensates (Messenger et al. 2003). Laboratory simulation of particle coagulation in the solar nebula suggests that particles grow under the ballistic cluster-cluster aggregation (BCCA) process (Wurm \& Blum 1998). The morphology of dust aggregates in comets reflects the formation and evolution history of cometary dust, which is far from being comprehensible. Nonetheless, it is reasonable to model cometary dust with BCCA particles in the first place, while we also consider particles grown under the ballistic particle-cluster aggregation (BPCA) process for comparison (see Kitada et al. 1993). The BPCA and BCCA particles consisting of identical spheres are known to be fractals described with the fractal dimension of $D \approx 3$ and 2, respectively (Meakin 1983, 1984). Because of the anti-correlation between fractal dimension and porosity at a fixed volume, BPCA particles are relatively compact compared to BCCA particles (Mukai et al. 1992). These aggregate particles can be regarded as two extreme cases among ballistic aggregation processes, the details of which are discussed in Blum et al. (1994).

It is worthwhile noting that the structures of these aggregates are similar to those of interplanetary dust particles (IDPs) collected in the stratosphere of the earth (cf. Brownlee 1985). In particular, BCCA particles represent the highly open structures of "cluster" IDPs well, which fragmented upon impact with the collector. Cluster IDPs are supposedly of cometary origin, owing to their pristine characteristics such as hydrogen and nitrogen isotopic anomalies and the presence of silicates with anomalous oxygen isotopic compositions (Messenger 2000; Messenger et al. 2003). It should be, however, noted that the choice of monomers' configuration is not crucial to our study, because light-scattering properties of aggregates do not strongly depend on their structures (see Kimura 2001; Kimura et al. 2003; Kimura \& Mann 2004).

Cometary dust might be partially and locally aligned as inferred from very faint circular polarization that varies within a coma (Dollfus \& Suchail 1987; Rosenbush et al. 1997; Manset \& Bastien 2000). However, random orientation is a reasonable assumption for cometary dust, when one considers global optical features in a whole coma. Our numerical results are, therefore, shown after being averaged over particle orientation with respect to the incident wave. Orientational averaging suffices to dilute any peculiarities in albedo and linear polarization for each aggregate consisting of submicron monomers (Kolokolova et al. 2006). For the numerical calculations given in Sect. 3, we use single BPCA and BCCA particles that consist of submicron monomers as determined in the next subsection.

\subsection{The size of monomers}

It may be sensible to seek information on monomer's size from IDPs that are currently the only samples of possibly cometary dust accessible to detailed laboratory analyses. Typical IDPs of aggregates consist of $100 \mathrm{~nm}$ sized grains, which are further composed of much smaller grains (Brownlee 1978). The constituent grains are certainly not monodisperse, but most of the mass appears to lie in the $100-500 \mathrm{~nm}$ size range (Brownlee et al. 1980). It should be noted that there is apparently a lack of information on the size distribution of the constituent grains. Here we simply ignore the size distribution of monomers, while 
its influence on the light-scattering properties of aggregates needs to be studied in future.

The size of the constituents forming aggregate IDPs does not contradict our understanding of cometary dust aggregates from a theoretical point of view. Greenberg \& Hage (1990) argue the presence of $100 \mathrm{~nm}$ grains as basic constituents of cometary dust aggregates to reproduce the ratio of the 3.4 and $9.7 \mu \mathrm{m}$ emission bands observed for comet 1P/Halley. In addition, aggregate particles with monomer's radius $a_{\mathrm{m}}=100 \mathrm{~nm}$ have so far been successful for modelling thermal emission spectra of comets (Greenberg \& Hage 1990; Li \& Greenberg 1998a,b; Greenberg \& Li 1999a). In this paper, we consider monomers around $100 \mathrm{~nm}$ in radius, more precisely, in the range of $100 \pm 30 \mathrm{~nm}$.

\subsection{The shape of monomers}

If monomers are small enough to interact with solar radiation in the Rayleigh scattering regime, the shape of the monomers does not play an important role. However, submicron monomers are expected to show deviation from the Rayleigh scattering in the visible wavelength range, and thus attention should be paid to the effect of the monomer's shape. Xing \& Hanner (1997) have shown numerically that the nonsphericity of $250 \mathrm{~nm}$-radius monomers weakens oscillations slightly in the angular dependence of linear polarization, but does not significantly influence the angular dependence of albedo. Microwave analog experiments on light scattering by aggregates have also demonstrated that the shape of submicronequivalent monomers causes only minor changes in the maximum polarization and does not influence the wavelength dependence of albedo (Gustafson \& Kolokolova 1999). Recent numerical calculations by Köhler et al. (2006) show that the single-scattering albedo and asymmetry parameter at a wavelength of $\lambda=600 \mathrm{~nm}$ do not strongly depend on the shape of monomers with $a_{\mathrm{m}}=100 \mathrm{~nm}$. Therefore, the light-scattering properties of aggregates consisting of non-spherical submicron monomers may not differ drastically from those of clusters of submicron spheres.

Imaging with scanning electron microscopes has revealed that the constituent grains of aggregate IDPs are fairly equidimensional and rounded (Brownlee et al. 1980). In this paper, we consider clusters of spherical monomers, because this not only simplifies the numerical configuration of the monomers but also provides a rigorous solution for light scattering by the clusters.

\subsection{The composition of monomers}

In a light-scattering theory, the complex refractive index $m$ is the quantity that describes the material composition of a particle. We first assume that the refractive index is independent of wavelength (Sect. 3.1) and second consider the refractive index that varies with wavelength (Sect. 3.2). This allows us to observe clearly how the spectral variation of refractive index affects the optical properties of aggregates. In this study, the wavelength-independent refractive index $m=n+\mathrm{i} k$ is taken in the range $n=1.4-2.0$ for the real part and $k=0.01-1.0$ for the imaginary part. This covers the value widely from optically transparent to dark particles that appear in previous models of cometary dust.

In a recent work, we consulted the composition of dust from comet $1 \mathrm{P} /$ Halley to estimate the average refractive index of cometary dust (Kimura et al. 2003). Impact-ionization mass spectra of 1P/Halley's dust measured in situ suggest that the dust consists of magnesium-rich silicates, carbonaceous materials, and iron-bearing sulfides (Jessberger et al. 1988; Jessberger 1999). These materials are also known to be major constituents of IDPs with inhomogeneity on a submicron scale (Brownlee et al. 1980; Jessberger et al. 2001). A study of light scattering by aggregate particles consisting of heterogeneous monomers is certainly desired, but this is beyond the scope of the present work. Here we consider aggregates of magnesium-rich silicate, those of carbonaceous material, those of iron-bearing sulfide, and those of a homogeneous mixture of these materials. For a mixture of materials, we estimate the volume fractions of magnesium-rich silicates, carbonaceous materials, and iron-bearing sulfides to be $27.7,66.3$, and $6.0 \%$, respectively, according to the composition of $1 \mathrm{P} /$ Halley's dust (cf. Mann et al. 2004). This leads to the mass ratio of carbonaceous materials to rock-forming materials being unity, which is consistent with the mass distribution of condensable elements in comets (Greenberg 1983).

Fomenkova et al. (1994) have proposed that the carbonaceous compounds of Halley's dust consist of pure carbon, hydrocarbons, carbonates, polymers, and complex organic matter (see, Fomenkova 1999). Li \& Greenberg (1998b) showed that amorphous carbon alone in the carbonaceous component is sufficient to reproduce the infrared spectra of $1 \mathrm{P} / \mathrm{Halley}$, while Greenberg \& $\operatorname{Li}$ (1999b) used organic refractory alone in the carbonaceous component to reproduce the infrared spectra of 19P/Borrelly. By taking similarities in the refractive indices of carbonaceous materials into account, we use amorphous carbon and organic refractory to simulate the carbonaceous components of cometary dust. Material processing of $1 \mathrm{P} /$ Halley's dust is compatible with the case that one third of the carbonaceous materials is organic refractory and two thirds amorphous carbon (Kimura et al. 2003; Kimura \& Mann 2006). Nevertheless, the difference in the infrared spectra of shortperiod comets between 1P/Halley and 19P/Borrelly may indicate that the ratio of amorphous carbon to organic refractory in cometary dust varies from one comet to another (Li \& Greenberg 1998b; Greenberg \& Li 1999b). Therefore, we take the volume fraction of organic refractory within the carbonaceous materials as a parameter.

Laboratory analyses of cluster IDPs show that organics and rock-forming materials are enclosed with amorphous carbon (Flynn et al. 1999; Keller et al. 2000). Supposing that amorphous carbon encases organic refractory, magnesium-rich silicate, and iron-bearing sulfide in the constituent particles of dust aggregates, we estimate effective refractive indices for such a mixture using the Maxwell-Garnett mixing rule (see Bohren \& Huffman 1983). Table 1 lists the wavelength-dependent refractive indices for magnesium-rich olivine, organic refractory, amorphous carbon, and pyrrhotite used in the calculations. 
Table 1. Complex refractive indices $(m=n+\mathrm{i} k$ ) at a wavelength $\lambda$ for candidate constituent materials.

\begin{tabular}{llll}
\hline \hline Material & $m$ at $\lambda=450 \mathrm{~nm}$ & $m$ at $\lambda=600 \mathrm{~nm}$ & Reference \\
\hline Magnesium-rich olivine & $1.69+\mathrm{i} 1.04 \times 10^{-4}$ & $1.68+\mathrm{i} 1.15 \times 10^{-5}$ & Huffman (1976) \\
Organic refractory & $1.91+\mathrm{i} 3.17 \times 10^{-1}$ & $1.97+\mathrm{i} 2.79 \times 10^{-1}$ & Li \& Greenberg (1997) \\
Amorphous carbon & $1.95+\mathrm{i} 7.86 \times 10^{-1}$ & $2.14+\mathrm{i} 8.05 \times 10^{-1}$ & Rouleau \& Martin (1991) \\
Pyrrhotite & $1.45+\mathrm{i} 1.53$ & $1.70+\mathrm{i} 1.86$ & Egan \& Hilgeman (1977) \\
\hline
\end{tabular}

Table 2. Complex refractive indices $(m=n+\mathrm{i} k)$ at a wavelength $\lambda$ for a mixture of carbonaceous materials, magnesium-rich silicate, and iron-bearing sulfide.

\begin{tabular}{lll}
\hline \hline$f_{\text {or }}{ }^{a}$ & $m$ at $\lambda=450 \mathrm{~nm}$ & $m$ at $\lambda=600 \mathrm{~nm}$ \\
\hline 0.00 & $1.85+\mathrm{i} 6.07 \times 10^{-1}$ & $1.98+\mathrm{i} 6.43 \times 10^{-1}$ \\
0.05 & $1.85+\mathrm{i} 5.92 \times 10^{-1}$ & $1.98+\mathrm{i} 6.26 \times 10^{-1}$ \\
0.10 & $1.84+\mathrm{i} 5.76 \times 10^{-1}$ & $1.97+\mathrm{i} 6.08 \times 10^{-1}$ \\
0.15 & $1.84+\mathrm{i} 5.61 \times 10^{-1}$ & $1.97+\mathrm{i} 5.91 \times 10^{-1}$ \\
0.20 & $1.84+\mathrm{i} 5.45 \times 10^{-1}$ & $1.96+\mathrm{i} 5.73 \times 10^{-1}$ \\
0.25 & $1.84+\mathrm{i} 5.30 \times 10^{-1}$ & $1.96+\mathrm{i} 5.56 \times 10^{-1}$ \\
0.30 & $1.84+\mathrm{i} 5.14 \times 10^{-1}$ & $1.95+\mathrm{i} 5.38 \times 10^{-1}$ \\
\hline
\end{tabular}

${ }^{a}$ The volume fraction of organic refractory occupying carbonaceous materials is given as $f_{\text {or }}$ and thus that of amorphous carbon is $1-f_{\text {or }}$.

Note that any realistic materials do have wavelength-dependent refractive indices, while the spectral variation may not be significant for some of the materials. Table 2 provides the effective refractive indices of the synthetic mixture as a function of the volume fraction $f_{\text {or }}$ of organic refractory within the carbonaceous materials in the range $f_{\text {or }}=0.0-0.3$.

\subsection{The number of monomers}

Volume $V$ of an aggregate consisting of $N$ spherical monomers is simply proportional to the number of monomers as $V=$ $(4 / 3) \pi a_{\mathrm{m}}^{3} N$. The radius of an aggregate particle can be described by the radius of a sphere of equal volume given as $a_{\mathrm{V}}=a_{\mathrm{m}} N^{1 / 3}$. Note that BPCA and BCCA particles are different in their visual sizes even if their radii of a volumeequivalent sphere are identical. An apparent size of an aggregate may be better characterized by the radius $a_{\mathrm{c}}=\sqrt{5 / 3} a_{\mathrm{g}}$, where $a_{\mathrm{g}}$ is the radius of gyration (see Mukai et al. 1992). If we define the porosity $P$ of an aggregate by $P=1-N\left(a_{\mathrm{m}} / a_{\mathrm{c}}\right)^{3}$, the BPCA and BCCA particles of $N=128$ used in the present study have $P=0.90$ and 0.94 , respectively.

We do not average the results over particle sizes to avoid increasing the number of model parameters such as minimum and maximum sizes and a size distribution function. While our computing resources could deal with up to $N=256$ or even $N=512$, depending on the other parameters, a large number $N$ results in a long computing time. We mainly concentrate our study on $N=128\left(a_{\mathrm{V}}=0.50 \mu \mathrm{m}\right.$ for $\left.a_{\mathrm{m}}=100 \mathrm{~nm}\right)$ to survey a wide range of parameters within a limited computation time. It should be noted, however, that this does not restrict our study, because the number of monomers does not strongly influence the light-scattering properties of aggregates (Kimura 2001).

\section{Numerical results}

Numerical calculations are performed by the superposition T-matrix method (TMM), which gives rigorous solutions for a cluster of homogeneous spheres (Mackowski \& Mishchenko 1996; Fuller \& Mackowski 2000). Alternatively, the solutions can be found by the generalized multisphere-Mie theory (GMM), which has been developed by Xu (1995), or can be estimated even by the discrete-diploe approximation (DDA), which has been developed by Draine \& Flatau (1994). The advantage of the T-matrix formalism is the orientation average of scattering matrix that can be performed analytically. Therefore, calculation with the superposition TMM code is fast, but is often limited to a small number $N$ of monomers unless huge random-access memory is allocated. We could use the T-matrix formalism of GMM by Xu (2003), which supposedly overcomes the limitation of $N$, or calculate the T-matrix of aggregates using DDA as shown by Mackowski (2002), but these possibilities are left for a future study.

We present our numerical results for albedo $A$ and linear polarization $P$ as functions of scattering angle $\theta$, wavelength $\lambda$, and complex refractive index $m$. The albedo is defined as $A=\left(S_{11} \lambda^{2}\right) /(4 \pi G)$, where $S_{11}$ denotes an orientationally averaged element of a $4 \times 4$ scattering matrix $S_{i j}(i, j=1-4)$ and $G$ is the geometric cross section of aggregates (cf. Hanner et al. 1981). We compute the effective surface area $4 G$ of aggregates using a Monte-Carlo method, the details of which are described in Kitada et al. (1993). The degree of linear polarization is calculated as $P=-S_{12} / S_{11}$, where $S_{12}$ is an orientationally averaged element of the scattering matrix (Bohren \& Huffman 1983). We consider two wavelengths $\lambda=450$ and $600 \mathrm{~nm}$ to study the spectral variations of albedo and polarization in the visible wavelength range.

\subsection{Wavelength-independent refractive indices}

Each panel of Fig. 1 shows our numerical results of albedo and polarization as a function of scattering angle. The number $N$ of spherical monomers is fixed to $N=128$ and the radius $a_{\mathrm{m}}$ of the identical monomers is here assumed to be $a_{\mathrm{m}}=90 \mathrm{~nm}$ $\left(a_{\mathrm{V}}=0.45 \mu \mathrm{m}\right)$. The imaginary part of refractive index is $k=0.01$ (top), $k=0.1$ (middle), or $k=1.0$ (bottom), while the real part of refractive index is increased from $n=1.4$ to 2.0 with 0.1 steps in each panel. Solid curves indicate the results for the BCCA particles at a wavelength $\lambda=450 \mathrm{~nm}$, and 

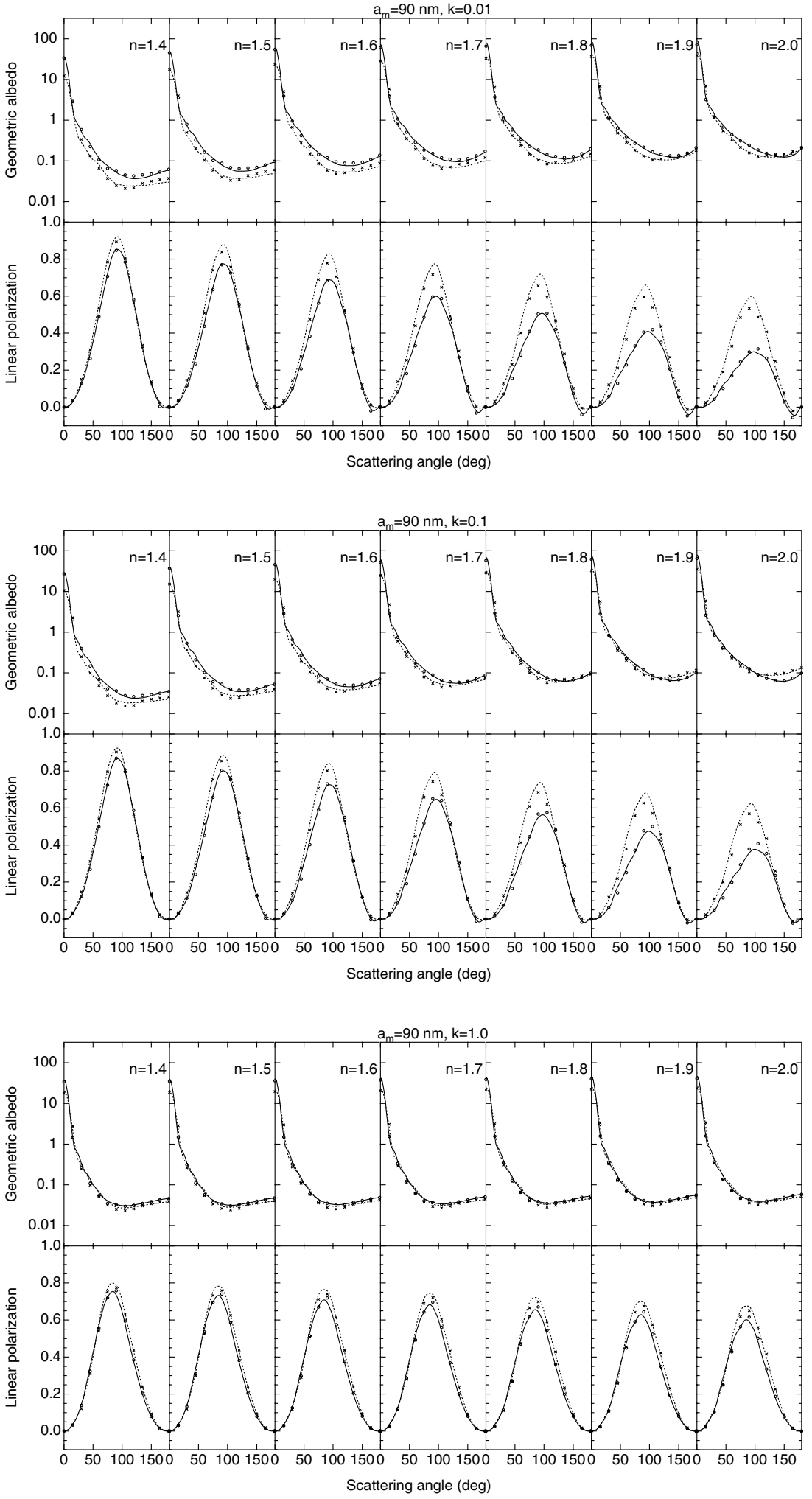

Fig. 1. Albedo and linear polarization as a function of scattering angle for aggregate particles consisting of 128 monomers with radius $a_{\mathrm{m}}=90 \mathrm{~nm}$ and the imaginary part of refractive index $k=0.01$ (upper panel), 0.1 (middle panel), and 1.0 (lower panel). Solid lines: results at a wavelength $\lambda=$ $450 \mathrm{~nm}$ for particles grown under the ballistic cluster-cluster aggregation (BCCA) process; dotted lines: results at $\lambda=600 \mathrm{~nm}$ for BCCA particles; open circles: results at $\lambda=$ $450 \mathrm{~nm}$ for particles grown under the ballistic particle-cluster aggregation (BPCA) process; crosses: results at $\lambda=600 \mathrm{~nm}$ for BPCA particles. dotted curves indicate those at $\lambda=600 \mathrm{~nm}$. The results for the BPCA particles are plotted as circles for $\lambda=450 \mathrm{~nm}$ and crosses for $\lambda=600 \mathrm{~nm}$ with an interval of $15^{\circ}$ over the results for the BCCA particles. Figures $2-4$ show the numerical results for monomers' radii $a_{\mathrm{m}}=100,110$, and $120 \mathrm{~nm}$, respectively. Figures 1 through 4 confirm the similarity in the optical properties of BCCA and BPCA particles, as already noticed in the previous works.

For all sizes of monomers considered here, the albedo increases with the real part $n$ of refractive index for aggregate particles having a low imaginary part $k$, but it is nearly independent of the real part at a high imaginary part. At a fixed 
value of the real part, an increase in the imaginary part lowers the albedo for the aggregates with a high real part, but this is not necessarily the case for those with a low real part. Therefore, the low albedo derived from observations cannot be obtained with a combination of a high real part and a low imaginary part. Proper angular dependences of albedo is often accompanied by a blue color unlike the red color observed for cometary dust.

In contrast with albedo, the polarization decreases as the real part of the refractive index increases, consistent with the results by Tishkovets et al. (2004). It is worth noting that this tendency is less pronounced for aggregate particles with a high imaginary part. When an aggregate has a low real part, the maximum polarization decreases with the imaginary part as noticed by Petrova et al. (2004). However, this tendency does not hold for a high real part where the maximum polarization increases with the imaginary part. In comparison with observations, the polarization shows either the maximum value as much higher than $10-30 \%$ or an angular dependence different from bellshaped. The polarization reaches its maximum at a scattering angle $\theta_{\max } \geq 90^{\circ}$ for a low imaginary part of the refractive index $k \leq 0.1$ and at $\theta_{\max } \leq 90^{\circ}$ for a high imaginary part $k=1.0$. Therefore, the observational constraint $\theta_{\max } \leq 90^{\circ}$ is fulfilled by aggregates consisting of optically dark material (i.e., $k>0.1$ ). An increase in the $n$-value at a fixed $k$-value tends to shift the $\theta_{\max }$-value toward a larger scattering angle for $k \leq 0.1$ and a smaller scattering angle for $k=1.0$. This means that the $\theta_{\max }$-value generally decreases with increase in the $k$-value at a fixed $n$-value. For $k \leq 0.1$, the negative branch of the polarization in the backscattering domain becomes obvious by increasing the $n$-values and lowering the $k$-values. However, the presence of negative polarization is associated with the scattering angle $\theta_{\max } \geq 90^{\circ}$, opposed to the $\theta_{\max }<90^{\circ}$ derived from observations. For $k=1.0$, the negative branch is absent for $a_{\mathrm{m}} \leq 100 \mathrm{~nm}$, but it becomes obvious for $a_{\mathrm{m}}>100 \mathrm{~nm}$ as $a_{\mathrm{m}}$ increases. However, the negative polarization very likely appears even for $a_{\mathrm{m}} \leq 100 \mathrm{~nm}$, if we consider overall sizes of aggregates much larger than $N=128$ (Nakamura \& Okamoto 1999; Kimura 2001).

In all the above cases, the angular and spectral dependences of the albedo and the polarization differ from the common optical characteristics observed for cometary dust. Nevertheless, we notice from Figs. 1 through 4 that the refractive index of $n \approx 1.8-2.0$ and $k \approx 0.1-1.0$ at $a_{\mathrm{m}} \approx 100 \mathrm{~nm}$ shows better results than the other combination of parameters. To seek the best combination(s) of the parameters, further calculations are performed within this range of the parameters. Note that the necessity of negative polarization in the backscattering region is not taken into account here, because we can attribute the presence of negative polarization to a consequence of larger overall sizes $N>128$. Figure 5 depicts the results at $a_{\mathrm{m}}=100 \mathrm{~nm}$ with $n=1.8$ (top), $n=1.9$ (middle), or $n=2.0$ (bottom) by changing the $k$-value from 0.2 to 0.8 with 0.1 steps. We find that satisfactory results can be obtained for aggregates with $a_{\mathrm{m}}=100 \mathrm{~nm}$ in the range of parameters $n=1.8-2.0, k=$ 0.4-0.6. This range of the parameters yields the common optical properties listed in the introduction except for the presence
Table 3. Summary of the results for albedo $A$ and polarization $P$ with wavelength-independent refractive index $(m=n+\mathrm{i} k)$ for aggregates of 128 monomers with radius $a_{\mathrm{m}}=100 \mathrm{~nm}^{a}$.

\begin{tabular}{lcccc}
\hline \hline$n$ & $k$ & $0.0-0.2$ & $0.4-0.6$ & $0.8-1.0$ \\
\hline $1.4-1.6$ & - & $A_{\theta} P_{\theta \lambda}$ & $A_{\theta} P_{\theta \lambda}$ \\
\hline $1.8-2.0$ & $P_{\lambda}$ & $A_{\theta \lambda} P_{\theta \lambda}$ & $A_{\theta} P_{\theta \lambda}$ \\
\hline
\end{tabular}

${ }^{a}$ The subscripts $\theta$ and $\lambda$ denote the successful retrievals of angular and spectral dependences, respectively.

of negative polarization and a quantitative fit to the maximum value of the polarization.

In Table 3, we summarize our numerical results for aggregate particles of 128 spherical monomers having radius $a_{\mathrm{m}}=100 \mathrm{~nm}$. A reasonable agreement between observations and model results in a certain range of refractive index is here indicated by $A_{\theta}$ for the angular variation of albedo, $A_{\lambda}$ for the spectral variation of albedo, $P_{\theta}$ for the angular variation of polarization, and $P_{\lambda}$ for the spectral variation of polarization.

\subsection{Wavelength-dependent refractive indices}

Figure 6 shows the numerical results of albedo and polarization for aggregates composed of magnesium-rich olivine as a function of scattering angle. We note that the results for magnesium-rich pyroxene and amorphous silicates are similar to those for magnesium-rich olivine. The radius $a_{\mathrm{m}}$ of identical monomers used in the computations covers 70 to $130 \mathrm{~nm}$ with a constant step of $10 \mathrm{~nm}$. The results show either a blue color of albedo $\mathrm{d} A / \mathrm{d} \lambda<0$ or a maximum polarization at a scattering angle $\theta_{\max }>90^{\circ}$, both of which are inconsistent with the optical properties of cometary dust. It is easy to infer from Fig. 6 that larger or smaller sizes of monomers do not improve the results. We find that there is no parameter space for silicate aggregates that simultaneously reproduces all the observed characteristics of light scattering by cometary dust.

Figures 7 and 8 show the numerical results for organic refractory and amorphous carbon, respectively. The observed characteristics of light scattering by cometary dust can be attained easily by aggregates of carbonaceous materials at $a_{\mathrm{m}} \approx$ $100 \mathrm{~nm}$. In particular, aggregates composed of amorphous carbon at $a_{\mathrm{m}} \approx 100 \mathrm{~nm}$ reproduce all the observed characteristics reasonably well. It should be mentioned that the condition $\theta_{\max }<90^{\circ}$ is not fulfilled by aggregates of organic refractory for $a_{\mathrm{m}}=100 \mathrm{~nm}$.

Numerical results of albedo and polarization for aggregate particles composed of pyrrhotite are shown in Fig. 9. These particles have higher albedo and lower polarization than those of carbonaceous materials. Although the spectral dependence of pyrrhotite aggregates are red, their angular dependences are different from those expected for cometary dust. We should mention that albedo and polarization of pure iron (no sulfur) aggregates show similar spectral and angular dependences.

Figures 6 through 9 illustrate that even the slight differences in the numerical values between the BCCA and BPCA become indistinguishably small for larger monomers, as previously 

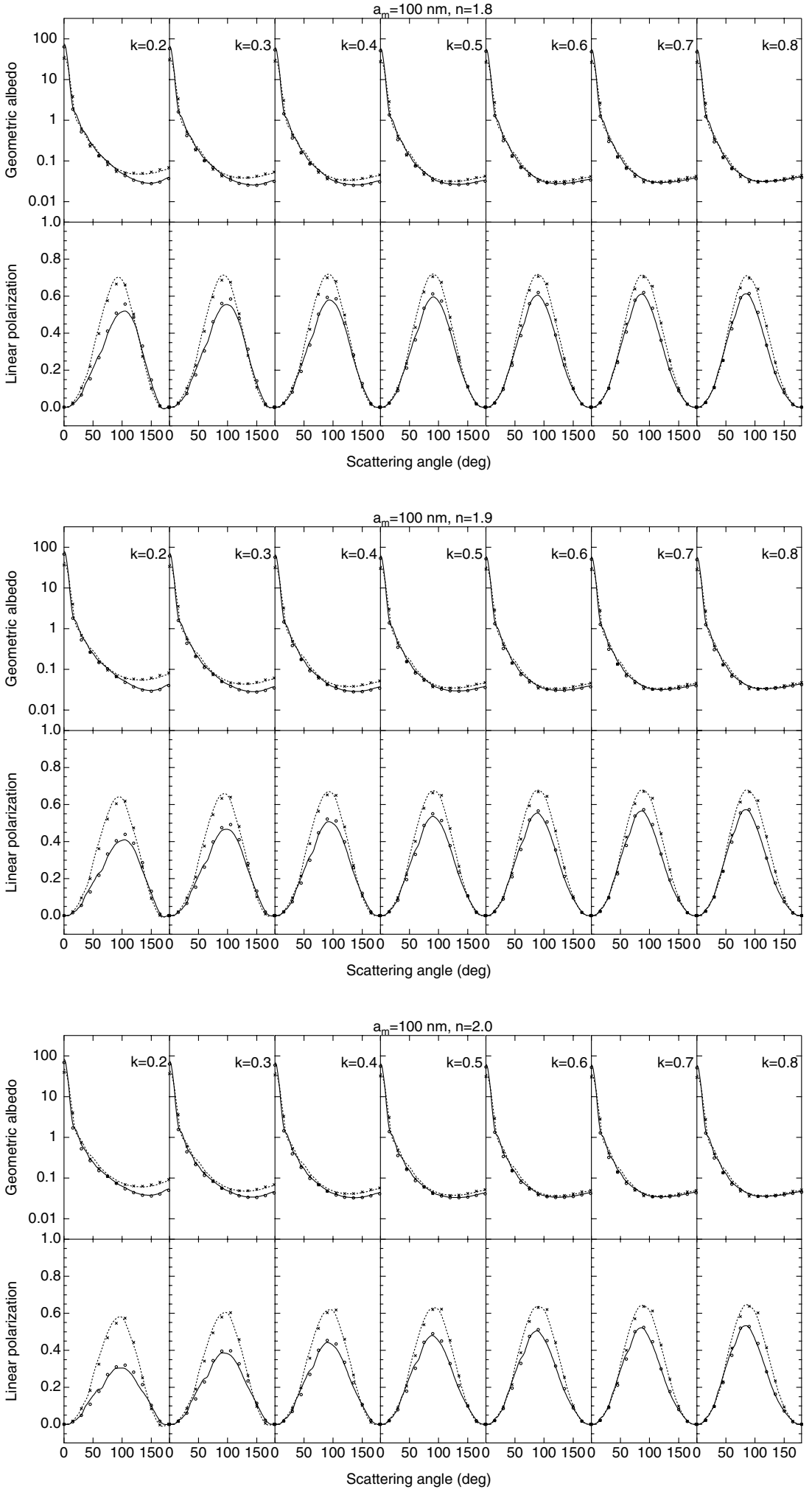

Fig. 5. Albedo and linear polarization as a function of scattering angle for an aggregate particle consisting of $N=128$ monomers with radius $a_{\mathrm{m}}=100 \mathrm{~nm}$ and the real part of refractive index $n=1.8$ (upper panel), $n=1.9$ (middle panel), and $n=2.0$ (lower panel). recognized (cf. Kimura 2001). Large sizes of monomers yield negative values in the polarization at the backscattering region, but they do not provide proper angular and spectral variations of the polarization.

Figure 10 displays the results with the synthetic mixture of magnesium-rich olivine, organic refractory, amorphous carbon, and pyrrhotite to simulate the average composition of cometary dust. The inferred composition of cometary dust is given as a function of a volume fraction $f_{\text {or }}$ of organic refractory in the range of $f_{\text {or }}=0.0-0.3$. Here the calculations are performed with a fixed number and radius of monomers: $N=128$ and $a_{\mathrm{m}}=100 \mathrm{~nm}\left(a_{\mathrm{V}}=0.5 \mu \mathrm{m}\right)$. The common optical characteristics of cometary dust are reproduced well not only by a mixture of silicate, organic refractory, amorphous carbon, and 

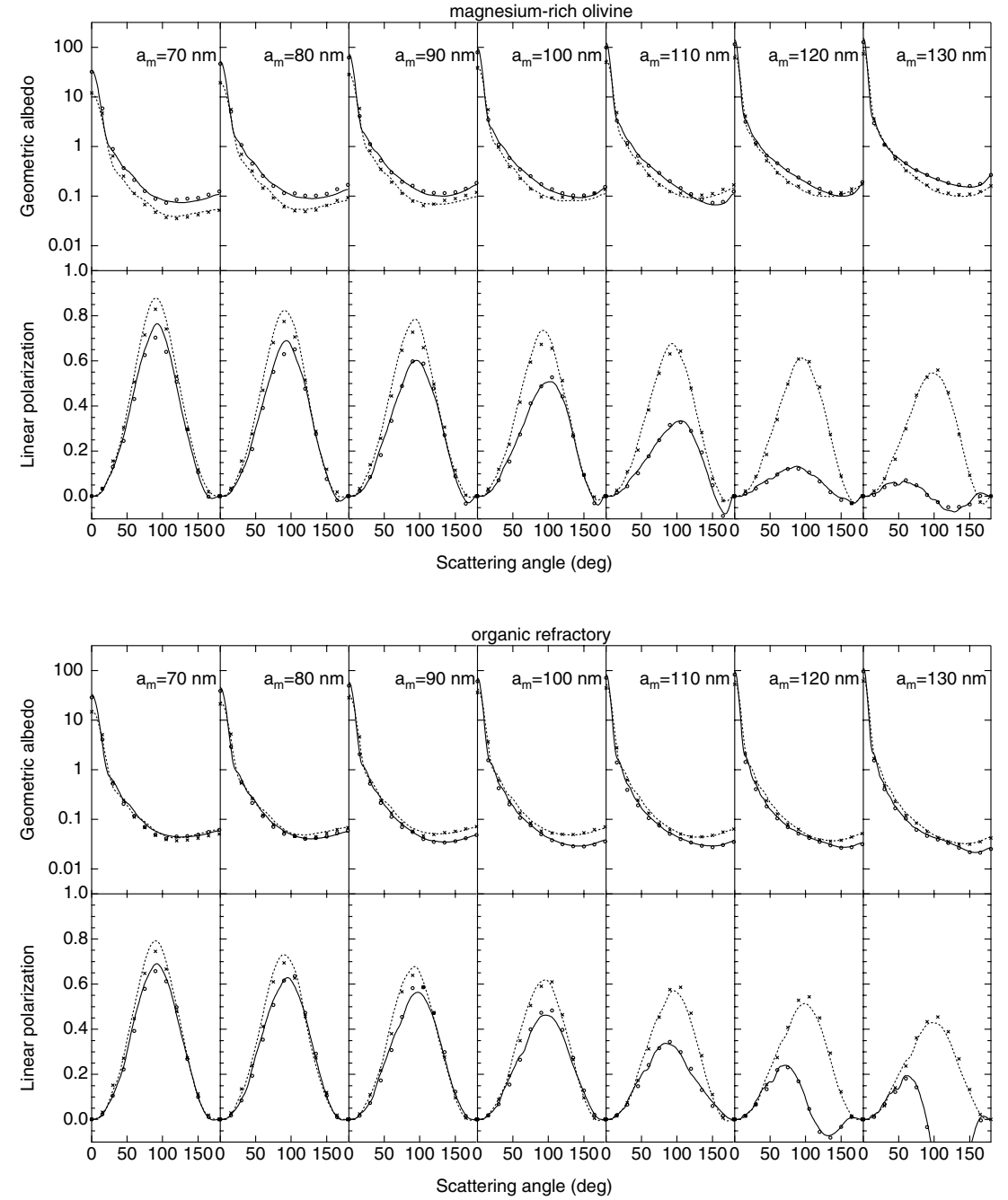

Fig. 6. The $N=128$ aggregate consisting of magnesium-rich olivine with the complex refractive index $m=1.69+\mathrm{i} 0.000104$ at a wavelength of $\lambda=450 \mathrm{~nm}$ and $m=1.68+\mathrm{i} 0.0000115$ at $\lambda=600 \mathrm{~nm}$

Fig. 7. The $N=128$ aggregate consisting of organic refractory with the complex refractive index $m=$ $1.91+\mathrm{i} 0.317$ at a wavelength of $\lambda=450 \mathrm{~nm}$ and $m=1.97+\mathrm{i} 0.279$ at $\lambda=600 \mathrm{~nm}$.

pyrrhotite, but also by a mixture excluding organic refractory (i.e., $f_{\text {or }}=0$ ). The results are insensitive to the amount of the organic refractory, while weak dependences of the polarization on the $f_{\text {or }}$ value may be worthy of mention. As the $f_{\text {or-value in- }}$ creases, the maximum polarization gradually increases and the scattering angle $\theta_{\max }$ of the maximum shifts from $\theta_{\max }<90^{\circ}$ to $\theta_{\max }>90^{\circ}$.

In Fig. 11, the results with $f_{\text {or }}=0$ are further shown at various sizes of aggregate particles, namely, in relation to the number $N$ of monomers. The albedo and polarization become nearly independent of the number of monomers for large aggregates $(N \geq 64)$. Small aggregates $(N<32)$ still show a bell-shaped polarization curve, but the albedo becomes more isotropic as $N$ decreases. The bottom panel of Fig. 11 illustrates the polarization curve at the backscattering region that is enlarged in the range $165 \leq \theta \leq 180^{\circ}$. It should be noted that, although not clear, the polarization at $N=256\left(a_{\mathrm{V}}=0.63 \mu \mathrm{m}\right)$ has a negative branch.

\section{Discussion}

\subsection{The size of monomers}

We have shown that the observed optical characteristics of cometary dust constrain the radius of monomers at $a_{\mathrm{m}} \approx 100 \mathrm{~nm}$ well. The optical properties of dust aggregates depend on the number of monomers in "neighborhood", which is marked out by a scale of $\lambda /(2 \pi)$ (Kimura \& Mann 2004). If the size parameter of monomers exceeds or is close to unity, then the number of neighbors is one or two, irrespective of the aggregate shape. Because optical observations indicate the independence of the albedo and polarization on the shape of aggregates, the size parameter of monomers in the optical wavelength range needs to be larger than unity, namely $a_{\mathrm{m}} \gtrsim 0.1 \mu \mathrm{m}$. The optimal radius of monomers $a_{\mathrm{m}} \approx 100 \mathrm{~nm}$ is thus consistent with the presence of uniqueness in the optical properties of cometary dust, while the value corresponds to the lower end of this constraint.

Near-infrared observations have shown that the negative polarization branch appears in $1 \mathrm{P} /$ Halley, but not in C/1995 O1 Hale-Bopp (Jones \& Gehrz 2000; Kelley et al. 2004). Petrova et al. (2004) speculate that this difference arises from the fact that dust aggregates in $1 \mathrm{P} /$ Halley contain larger monomers and/or have lower porosity than those in C/1995 O1 Hale-Bopp. We could attribute the difference to the porosity of aggregates, but not to the size of monomers: our results show that optical observations are compatible with our model of aggregate particles having the same sizes of monomers for different comets. The difference simply implies that the 

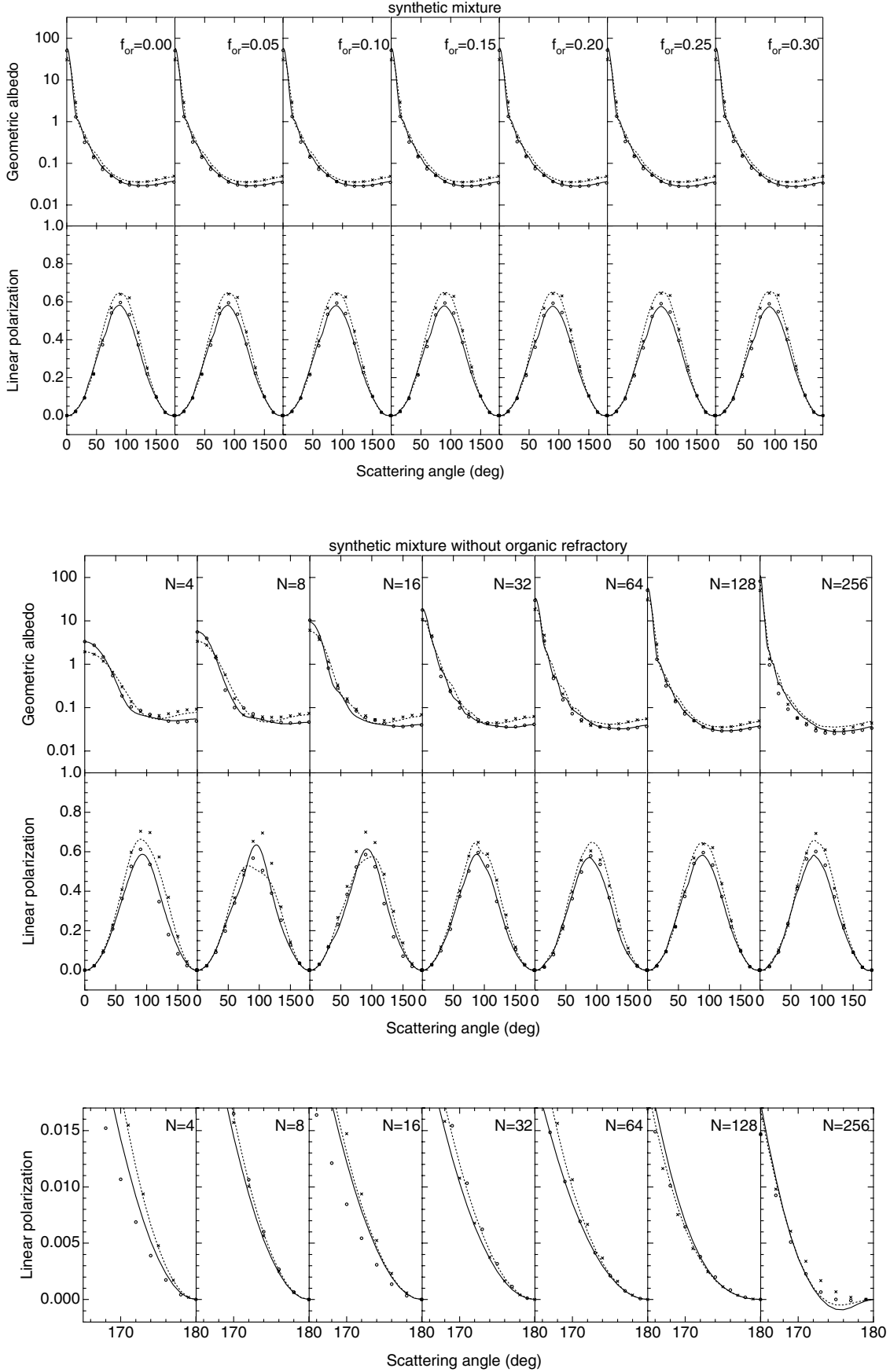

Fig. 10. Albedo and linear polarization as a function of scattering angle for aggregate particles of 128 monomers composed of a synthetic mixture of magnesium-rich olivine, organic refractory, amorphous carbon, and pyrrhotite. The results are shown with various assumptions for the volume fraction $f_{\text {or }}$ of organic refractory occupying carbonaceous materials.
Fig. 11. Albedo and linear polarization as a function of scattering angle for aggregate particles composed of a synthetic mixture of amorphous olivine, amorphous carbon, and pyrrhotite (top). The number of monomers increases from $N=4$ (left) to 256 (right) by doubling the monomer number. The backscatter region for the polarization curve is enlarged in the range of scattering angle between 165 and $180^{\circ}$. distance between two monomers, in other words, the diameter of monomers is smaller than the neighborhood scale of $\lambda /(2 \pi)$ in the near-infrared wavelength range. Therefore, near-infrared observations would place constraints on the upper-limit radius of monomers, such as $a_{\mathrm{m}}<0.2 \mu \mathrm{m}$ from observations at a wavelength of $\lambda=2.2 \mu \mathrm{m}$. All the available observations from optical to near-infrared wavelengths are consistent with the monomer radius of $a_{\mathrm{m}} \approx 100 \mathrm{~nm}$.

\subsection{The composition of monomers}

We have found that the common optical properties of cometary dust can be reproduced with a combination of the parameters $n \approx 1.8-2.0$ and $k \approx 0.4-0.6$ at $a_{\mathrm{m}} \approx 100 \mathrm{~nm}$, if the refractive index is independent of wavelength (Figs. 1-5). These refractive indices are close to the values for carbonaceous materials, but not to the values for silicates or sulfides (see Table 1). We should also note that the preferential values do not match the refractive indices of either metals or ices whose values are too high and too low, respectively (cf. Johnson \& Christy 1974; Warren 1984). In fact, our results with wavelength-dependent refractive indices show that aggregates composed of carbonaceous materials alone can reproduce the common optical characteristics of cometary dust well (Figs. 7 and 8). There is no range of parameters for aggregate particles consisting of silicates or sulfides to yield satisfactory results (Figs. 6 and 9). 
Our results with both wavelength-independent and wavelengthdependent refractive indices point to the predominance of carbonaceous materials in cometary dust. The arguments of condensable elements in comets also indicate that carbonaceous materials are the dominant component of cometary dust (Greenberg 1983; Huebner 2002). In addition, Halley's dust and aggregate IDPs are rich in carbonaceous materials, while they contain silicates and sulfides (Kissel et al. 1986; Jessberger et al. 1988; Thomas et al. 1993; Keller et al. 2000). The optically very dark appearances of aggregate IDPs have thus been attributed to the high carbon content (Brownlee 1978, 1985). Accordingly, we expect that the refractive indices of cometary dust resemble those of carbonaceous materials and that the other materials such as silicates and sulfides play only a minor role in determining the refractive indices.

All the optical properties of cometary dust listed in Sect. 1 are best reproduced with the synthetic mixture of carbonaceous materials together with silicate and sulfide in submicron grains forming a large aggregate particle (Figs. 10 and 11). The optimum parameters for aggregate particles consisting of the synthetic mixture agree well with the case for the wavelength-independent refractive index (cf. Tables 2 and 3). The imaginary part of the refractive indices for the synthetic mixture nevertheless lies slightly outside the range $k \approx 0.4-0.6$. Therefore, the wavelength-dependent nature of refractive index, especially, its positive spectral gradient (i.e., $\mathrm{d} m / \mathrm{d} \lambda>0$ ) relaxes the constraints on the parameter space, which provides satisfactory results.

\subsection{The number of monomers}

The present model with a cluster of spheres is successful, but needs refinement to quantitatively reproduce the angular dependence of linear polarization observed for cometary dust. The polarization at larger sizes of aggregates (i.e., $N>256$ ) is expected to have a lower maximum and a deeper and wider negative branch (Kimura \& Mann 2004). Our results also show for the mixture of amorphous carbon, magnesium-rich olivine, and iron-bearing sulfide that the negative polarization branch appears only at the largest size $N=256$, namely $a_{\mathrm{V}}=0.63 \mu \mathrm{m}$ (Fig. 11). Therefore, all the common optical characteristics of cometary dust might be quantitatively reproduced once larger aggregates could be dealt with in the future.

Besides, the spherical shape of the monomers might be the assumption that prevents us from reaching quantitative agreement with observed polarization. The physical and chemical properties of monomers have been known to influence the phase-angle dependence of polarization (West 1991; Xing \& Hanner 1997; Mann et al. 2004). Nonspherical monomers may lower the maximum polarization, because the polarization for spheroidal particles of a tenth part of micron is lower than that for spherical particles of equal volume (Mishchenko 1992). As an extension of the present work, the non-sphericity in the shape of monomers might be considered for calculation of light scattering by large aggregates.

\subsection{Processing of pristine materials}

The pristine monomers of dust aggregates in comets may consist of an amorphous silicate core and an organic refractory mantle (Greenberg \& Hage 1990; Kimura \& Mann 2004). A detailed study of the form of each material is important, because this could hint at the evolution of cometary materials. Processing is a known mechanism to transform organic refractory to amorphous carbon contained in the carbonaceous material of cometary dust (Jenniskens et al. 1993; Li \& Greenberg 1998b). Slight variations in the albedo and polarization data for a variety of comets may be interpreted in terms of the processing of cometary matter. The amount of organic refractory relative to amorphous carbon can be used as a measure of processing in the cometary matter (Greenberg \& Li 1999b; Kimura $\&$ Mann 2006). Our inspection of $\theta_{\max }$, the scattering angle for the maximum polarization, suggests that aggregate particles with a lesser amount of organic refractory provide better results than those with a higher amount of organic refractory material (Fig. 10). However, this might be an artifact if the $\theta_{\max }$ value decreases with increasing the number of monomers, as claimed by Petrova et al. (2004). In addition, there are large uncertainties as to whether organic refractory and amorphous carbon in cometary dust are simulated well by the man-made materials. The refractive index of carbonaceous material derived from laboratory measurements varies from one sample to another, depending on the physical and chemical properties of the sample prepared in the laboratory (Rouleau \& Martin 1991; Jenniskens 1993). Therefore, the current results with only one pair of refractive indices do not allow us to draw a firm conclusion about the degree of processing.

\subsection{Remarks on cometary dust models}

A number of authors have attempted to model optical properties of cometary dust using only the refractive indices of silicates (e.g., Mukai et al. 1987; Levasseur-Regourd et al. 1997; Petrova et al. 2000; Kerola \& Larson 2001). In particular, the presence of negative polarization in the backscatter domain has commonly been attributed to silicate or dirty ice grains. It is worth noting that a negative polarization branch could also appear in the angular dependences of linear polarization for large aggregates composed of absorbing materials (Lumme \& Rahola 1994; Haudebourg et al. 1999; Kimura 2001). In a previous study, silicate aggregates consisting of spherical monomers with $a_{\mathrm{m}}=150 \mathrm{~nm}$ provided quantitatively excellent results on the maximum and negative degrees of linear polarization at a single wavelength $\lambda=600 \mathrm{~nm}$, but carbon aggregates with the same parameters failed (Kimura 2001). Nevertheless, once the spectral dependences of albedo and polarization are taken into account, the results with such silicate aggregates are no longer consistent with observations (cf. Fig. 6). This clearly demonstrates the case that even a quantitative fit of numerical results to one of the observed features is insufficient for modelling cometary dust. We emphasize that a correct understanding of light scattering by cometary dust needs a model that simultaneously reproduces all the optical characteristics (see Kolokolova et al. 2004a,b). Interpretations 
of observational data based on a silicate-dust model that does not fulfill this requirement can be misleading.

The presence of silicates in cometary dust has been confirmed unambiguously by infrared observations of silicate features (e.g., Hanner 1999). However, the findings of infrared silicate features do not necessarily mean that silicates are the dominant component of the dust. The infrared silicate features observed for Halley's dust can be reasonably reproduced if a silicate component of an aggregate occupies one third of its total volume and an organic component fills the rest of the volume (Greenberg et al. 1996). This infrared modeling is supplementary to our comprehensive survey of parameters in the optical wavelength range. Both results strengthen the validity of our best model of cometary dust that imitates Halley's dust compositionally and aggregate IDPs morphologically. We conclude that all available information on the composition and structure of cometary dust is consistent and naturally directs us toward the most realistic model.

Acknowledgements. We would like to thank an anonymous referee for his/her helpful suggestions. We are indebted to Daniel Mackowski, Kirk Fuller, and Michael Mishchenko, who made their TMM code publicly available. This research is supported by the Ministry of Education, Culture, Sports, Science, and Technology MEXT (Monbu Kagaku Sho) under Grant-in-Aid for Scientific Research on Priority Areas "Development of Extra-Solar Planetary Science" (\#16077203), NASA Planetary Atmospheres Program, and the German Aerospace Center $D L R$ (Deutschen Zentrum für Luft- und Raumfahrt) under the project "Rosetta: MIDAS, MIRO, MUPUS" (RD-RX-50 QP 0403).

\section{References}

Blum, J., Henning, T., Ossenkopf, V., et al. 1994, in Fractals in the Natural and Applied Sciences, ed. M. M. Novak (Amsterdam: North-Holland), 47

Bohren, C. F., \& Huffman, D. R. 1983, Absorption and Scattering of Light by Small Particles (New York: Wiley-Interscience)

Brownlee, D. E. 1978, in Cosmic Dust, ed. J. A. M. McDonnell (New York: Wiley-Interscience), 295

Brownlee, D. E. 1985, Ann. Rev. Earth Planet. Sci., 13, 147

Brownlee, D. E., Pilachowski, L., Olszewski, E., \& Hodge, P. W. 1980, in Solid Particles in the Solar System, ed. I. Halliday, \& B. A. McIntosh (Dordrecht: D. Reidel), 333

Chernova, G. P., Kiselev, N. N., \& Jockers, K. 1996, Icarus, 103, 144 Dobrovolsky, O. V., Kiselev, N. N., \& Chernova, G. P. 1986, Earth Moon Planets, 34, 189

Dollfus, A., \& Suchail, J.-L. 1987, A\&A, 187, 669

Dollfus, A., Bastien, P., Le Borgne, J.-F., Levasseur-Regourd, A. C., \& Mukai, T. 1988, A\&A, 206, 348

Draine, B. T., \& Flatau, P. J. 1994, J. Opt. Soc. Am. A, 11, 1491

Eaton, N., Scarrott, S. M., \& Gledhill, T. M. 1992, MNRAS, 258, 384

Egan, W. G., \& Hilgeman, T. 1977, Icarus, 30, 413

Flynn, G. J., Keller, L. P., Jacobsen, C., \& Wirick, S. 1999, Lunar Planet. Sci., 30, 1091

Fomenkova, M. N. 1999, Space Sci. Rev., 90, 109

Fomenkova, M. N., Chang, S., \& Mukhin, L. M. 1994, Geochim. Cosmochim. Acta, 58, 4503

Fuller, K. A., \& Mackowski, D. W. 2000, in Light Scattering by Nonspherical Particles, ed. M. I. Mishchenko, J. W. Hovenier, \& L. D. Travis (San Diego: Academic Press), 225
Gehrz, R. D., \& Ney, E. P. 1992, Icarus, 100, 162

Giese, R. H. 1980, in Solid Particles in the Solar System, ed. I. Halliday, \& B. A. McIntosh (Dordrecht: D. Reidel), 1

Greenberg, J. M. 1983, in Comets, ed. L. L. Wilkening (Tucson: Univ. of Arizona Press), 131

Greenberg, J. M., \& Gustafson, B. Å. S. 1981, A\&A, 93, 35

Greenberg, J. M., \& Hage, J. I. 1990, ApJ, 361, 260

Greenberg, J. M., \& Li, A. 1999a, Planet. Space Sci., 47, 787

Greenberg, J. M., \& Li, A. 1999b, Space Sci. Rev., 90, 149

Greenberg, J. M., Li, A., Yamamoto, T., \& Kozasa, T. 1996, in Physics, Chemistry, and Dynamics of Interplanetary Dust, ed. B. A. S. Gustafson, \& M. S. Hanner (San Francisco: Astronomical Society of the Pacific Press), 497

Gustafson, B. Å. S., \& Kolokolova, L. 1999, J. Geophys. Res., 104, 31711

Hadamcik, E., Renard, J. B., Worms, J. C., Levasseur-Regourd, A. C., \& Masson, M. 2002, Icarus, 155, 497

Hanner, M. S. 1999, Space Sci. Rev., 90, 99

Hanner, M. S., Newburn, R. L. 1989, AJ, 97, 254

Hanner, M. S., Giese, R. H., Weiss, K., \& Zerull, R. 1981, A\&A, 104, 42

Haudebourg, V., Cabane, M., \& Levasseur-Regourd, A.-C. 1999, Phys. Chem. Earth C, 24, 603

Huebner, W. F. 2002, Earth Moon Planets, 89, 179

Huffman, D. R. 1976, in Solid State Astrophysics, ed. N. C. Wickramasinghe, \& D. J. Morgan (Dordrecht: D. Reidel), 191

Jenniskens, P. 1993, A\&A, 274, 653

Jenniskens, P., Baratta, G. A., Kouchi, A., et al. 1993, A\&A, 273, 583

Jessberger, E. K. 1999, Space Sci. Rev., 90, 91

Jessberger, E. K., Christoforidis, A., \& Kissel, J. 1988, Nature, 332, 691

Jessberger, E. K., Stephan, T., Rost, D., et al. 2001, in Interplanetary Dust, ed. E. Grün, B. A. S. Gustafson, S. F. Dermott, \& H. Fechtig (Heidelberg: Springer-Verlag), 253

Jewitt, D., \& Meech, K. J. 1986, ApJ, 310, 937

Johnson, P. B., \& Christy, R. W. 1974, Phys. Rev. B, 9, 5056

Jones, T. J., \& Gehrz, R. D. 2000, Icarus, 143, 338

Keller, L. P., Messenger, S., \& Bradley, J. P. 2000, J. Geophys. Res., 105,10397

Kelley, M. S., Woodward, C. E., Jones, T. J., Reach, W. T., \& Johnson, J. 2004, AJ, 127, 2398

Kerola, D. X., \& Larson, S. M. 2001, Icarus, 149, 351

Kikuchi, S., Mikami, Y., Mukai, T., Mukai, S., \& Hough, J. H. 1987, A\&A, 187, 689

Kimura, H. 2001, J. Quant. Spec. Radiat. Transf., 70, 581

Kimura, H., \& Mann, I. 2004, J. Quant. Spec. Radiat. Transf., 89, 155

Kimura, H., \& Mann, I. 2006, in Highlights of Astronomy, Vol. 13, ed. O. Engvold (San Francisco: Astronomical Society of the Pacific Press), in press

Kimura, H., Kolokolova, L., \& Mann, I. 2003, A\&A, 407, L5

Kiselev, N. N., \& Velichko, F. P. 1998, Icarus, 133, 286

Kissel, J., Sagdeev, R. Z., Bertaux, J. L., et al. 1986, Nature, 321, 280

Kitada, Y., Nakamura, R., \& Mukai, T. 1993, in The Third International Congress on Optical Particle Sizing, ed. M. Maeda (Yokohama: Keio Univ.), 121

Köhler, M., Kimura, H., \& Mann, I. 2006, A\&A, 448, 395

Kolokolova, L., \& Jockers, K. 1997, Planet. Space Sci., 45, 1543

Kolokolova, L., Jockers, K., Chernova, G., \& Kiselev, N. 1997, Icarus, 126,351

Kolokolova, L., Hanner, M. S., Levasseur-Regourd, A.-C., \& Gustafson, B. Å. S. 2004a, in Comets II, ed. M. Festou, U. Keller, $\&$ H. Weaver (Tucson: Univ. of Arizona), 577 
Kolokolova, L., Kimura, H., \& Mann, I. 2004b, in Photopolarimetry in Remote Sensing, ed. G. Videen, Y. Yatskiv, \& M. Mishchenko (Dordrecht: Kluwer Acad. Publ.), 431

Kolokolova, L., Kimura, H., Ziegler, K., \& Mann, I. 2006, J. Quant. Spec. Radiat. Transf., in press

Lamy, P. L., Malburet, P., Llebaria, A., \& Koutchmy, S. 1989, A\&A, 222,316

Levasseur-Regourd, A. C., Hadamcik, E., \& Renard, J. B. 1996, A\&A, 313,327

Levasseur-Regourd, A. C., Cabane, M., Worms, J. C., \& Haudebourg, V. 1997, Adv. Space Res., 20, (8) 1585

Li, A., \& Greenberg, J. M. 1997, A\&A, 323, 566

Li, A., \& Greenberg, J. M. 1998a, ApJ, 498, L83

Li, A., \& Greenberg, J. M. 1998b, A\&A, 338, 364

Lisse, C. 2002, Earth, Moon, Planets, 90, 497

Lumme, K., \& Rahola, J. 1994, ApJ, 425, 653

Lumme, K., Rahola, J., \& Hovenier, J. W. 1997, Icarus, 126, 455

Mackowski, D. W. 2002, J. Opt. Soc. Am. A, 19, 881

Mackowski, D. W., \& Mishchenko, M. I. 1996, J. Opt. Soc. Am. A, 13,2266

Mann, I., Kimura, H., \& Kolokolova, L. 2004, J. Quant. Spec. Radiat. Transf., 89, 291

Manset, N., \& Bastien, P. 2000, Icarus, 145, 203

Meakin, P. 1983, J. Colloid Interface Sci., 96, 415

Meakin, P. 1984, Phys. Rev. A, 29, 997

Messenger, S. 2000, Nature, 404, 968

Messenger, S., Keller, L. P., Stadermann, F. J., Walker, R. M., \& Zinner, E. 2003, Science, 300, 105
Michalsky, J. J. 1981, Icarus, 47, 388

Millis, R. L., A'Hearn, M. F., \& Thompson, D. T. 1982, AJ, 87, 1310

Mishchenko, M. I. 1992, Earth Moon Planets, 57, 203

Mukai, S., \& Mukai, T. 1990, Icarus, 86, 257

Mukai, T., \& Fechtig, H. 1983, Planet. Space Sci., 31, 655

Mukai, T., Mukai, S., \& Kikuchi, S. 1987, A\&A, 187, 650

Mukai, T., Ishimoto, H., Kozasa, T., Blum, J., \& Greenberg, J. M. 1992, A\&A, 262, 315

Muñoz, O., Volten, H., de Haan, J. F., Vassen, W., \& Hovenier, J. W. 2000, A\&A, 360, 777

Nakamura, R., \& Okamoto, H. 1999, Adv. Space Res., 23(7), 1209

Petrova, E. V., Jockers, K., \& Kiselev, N. N. 2000, Icarus, 148, 526

Petrova, E. V., Tishkovets, V. P., \& Jockers, K. 2004, Sol. System Res., 38, 309

Rosenbush, V. K., Shakhovskoj, N. M., \& Rosenbush, E. 1997, Earth Moon Planets, 78, 381

Rouleau, F., \& Martin, P. G. 1991, ApJ, 377, 526

Thomas, K. L., Blanford, G. E., Keller, L. P., Klöck, W., \& McKay, D. S. 1993, Geochim. Cosmochim. Acta, 57, 1551

Tishkovets, V. P., Petrova, E. V., \& Jockers, K. 2004, J. Quant. Spec. Radiat. Transf., 86, 241

Warren, S. G. 1984, Appl. Opt., 23, 1206

West, R. A. 1991, Appl. Opt., 30, 5316

Wurm, G., \& Blum, J. 1998, Icarus, 132, 125

Xing, Z., \& Hanner, M. S. 1997, A\&A, 324, 805

Xu, Y.-l. 1995, Appl. Opt., 34, 4573 (erratum, 1998, 37, 6494)

Xu, Y.-1. 2003, Phys. Rev. E, 67, 046620 


\section{Online Material}


H. Kimura et al.: Light scattering by cometary dust, Online Material p 2
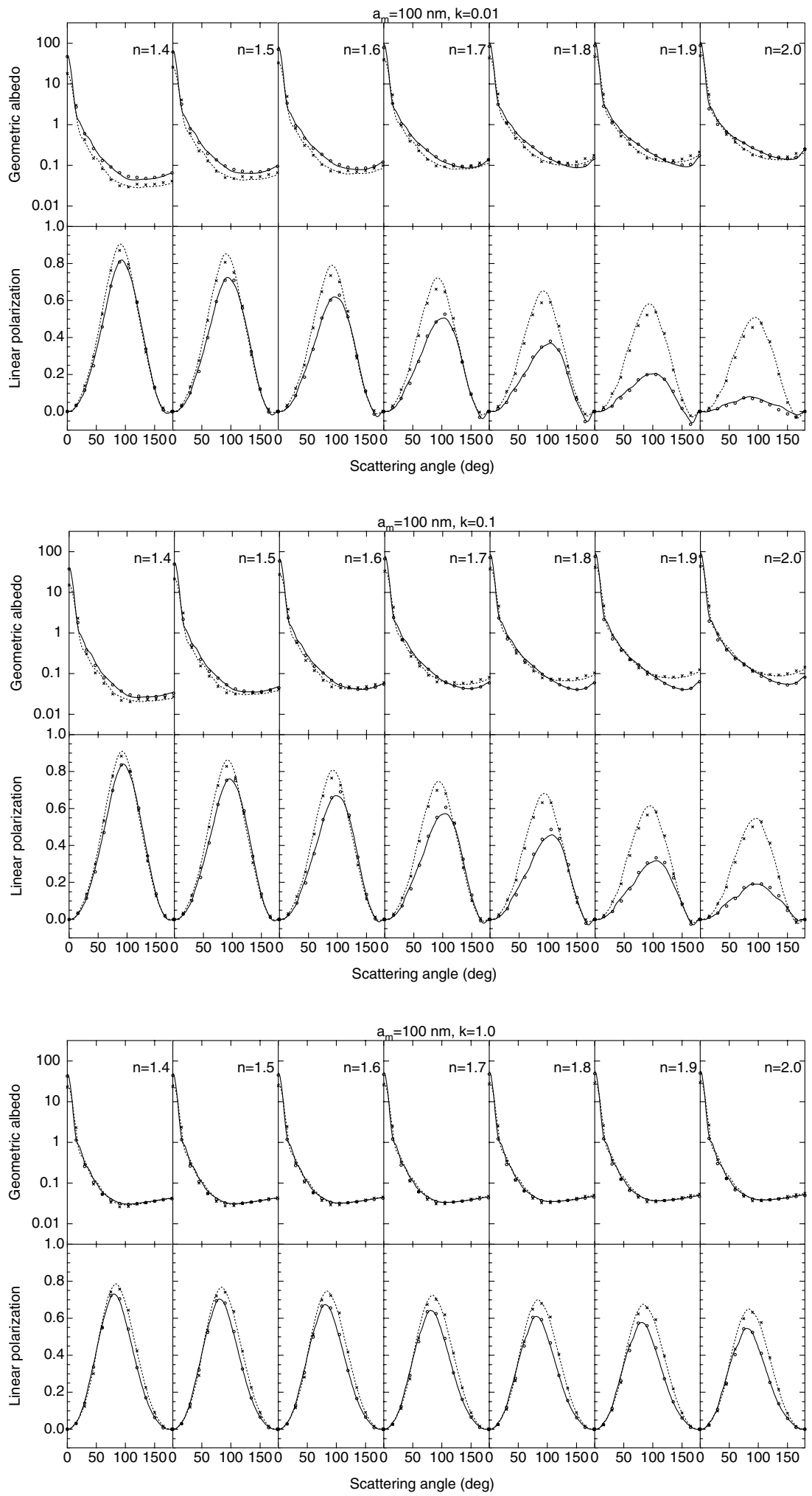

Fig. 2. The same as Fig. 1, but with radius $a_{\mathrm{m}}=100 \mathrm{~nm}$. 
H. Kimura et al.: Light scattering by cometary dust, Online Material $p 3$
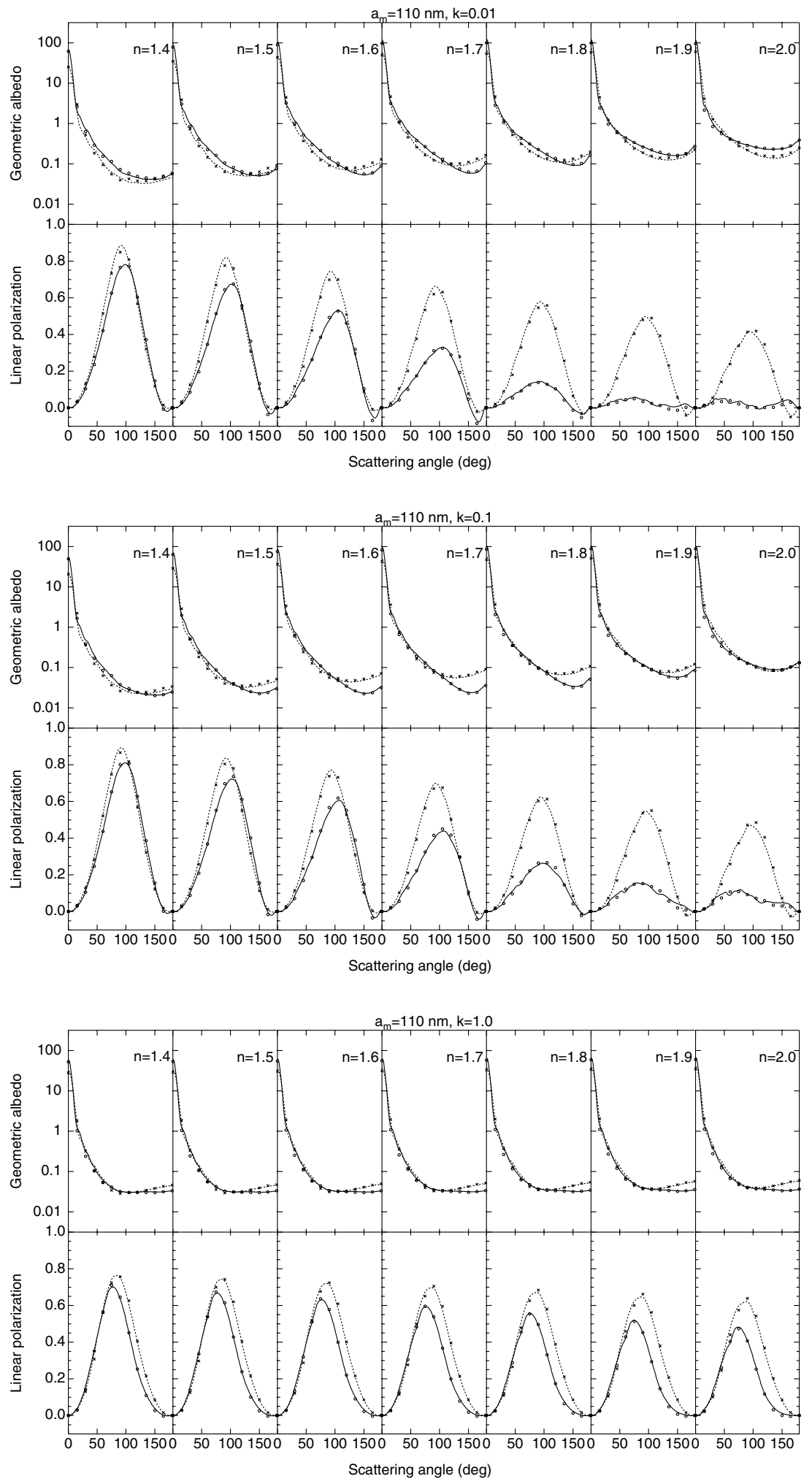

Fig. 3. The same as Fig. 1, but with radius $a_{\mathrm{m}}=110 \mathrm{~nm}$. 
H. Kimura et al.: Light scattering by cometary dust, Online Material p 4
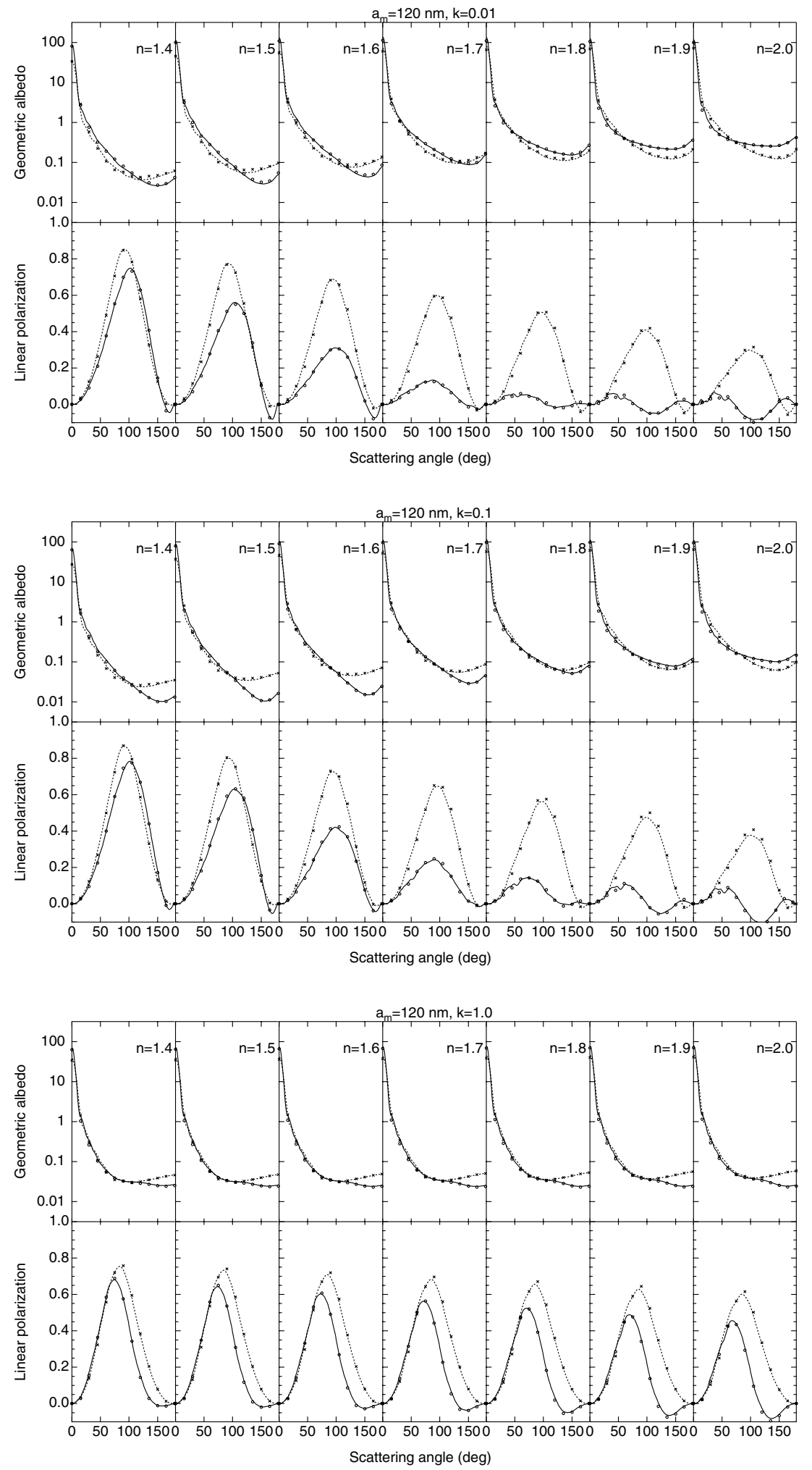

Fig. 4. The same as Fig. 1, but with radius $a_{\mathrm{m}}=120 \mathrm{~nm}$. 
H. Kimura et al.: Light scattering by cometary dust, Online Material p 5
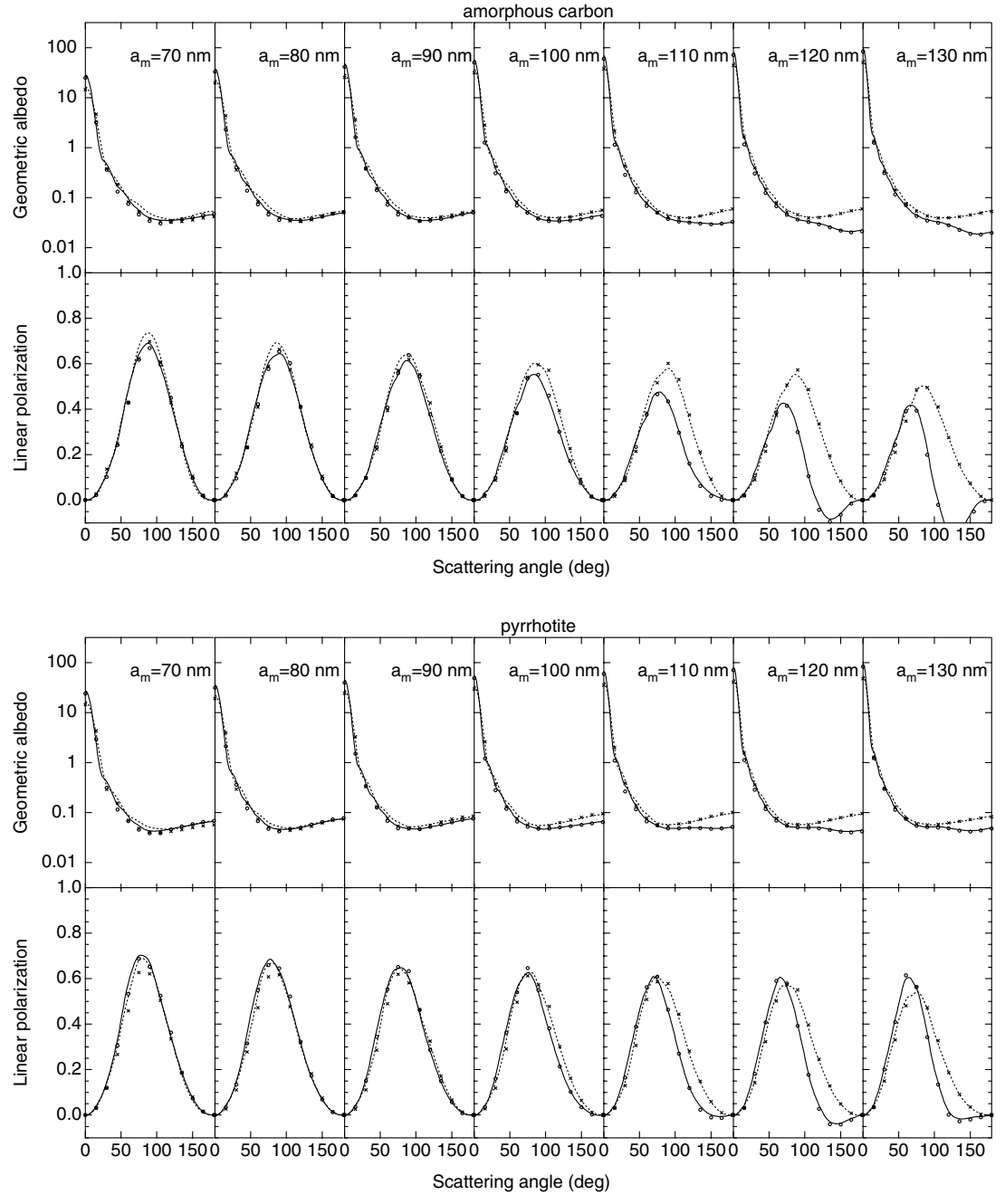

Fig. 8. The $N=128$ aggregate consisting of amorphous carbon with the complex refractive index $m=1.95+\mathrm{i} 0.786$ at a wavelength of $\lambda=450 \mathrm{~nm}$ and $m=2.14+\mathrm{i} 0.805$ at $\lambda=600 \mathrm{~nm}$.

Fig. 9. The $N=128$ aggregate consisting of pyrrhotite with the complex refractive index $m=$ $1.45+\mathrm{i} 1.53$ at a wavelength of $\lambda=450 \mathrm{~nm}$ and $m=1.70+\mathrm{i} 1.86$ at $\lambda=600 \mathrm{~nm}$. 